\title{
Voltage Stability Assessment using Artificial Neural Networks
}

\author{
K. Selvakumar*, C. S. Boopathi and M. Sri Harsha \\ Department of EEE, SRM University, Kattankulathur, Chennai - 603203,Tamil Nadu, India; selvakse@gmail.com, \\ cs.bhoopathy@gmail.com,mogilisriharsha@gmail.com
}

\begin{abstract}
Objectives: To calculate the load flow analysis by using Artificial Neural Networks (ANN) and the Cascade Architecture (CC) with Levenberg-Marquardt (LM) algorithm is used for this proposed system. Methods/Statistical Analysis: Many conventional methods such as Newton-Raphson method, Gauss-Seidel method, AC load flow analysis etc., are used to estimate the load flow analysis of a power system. The major backdrops in using these methods are, using complex non-linear equations, iterative methods and time consuming. To overcome these problems, this paper discusses using Artificial Neural Networks (ANN) which reduces the time consumption in calculating load flow analysis. Findings: In the real-time planning and operation of a power system the major consideration is voltage stability assessment. The voltage instability in a power system will lead to a blackout condition. The continuous increase in load demand, changes in system conditions causes voltage collapse. So the on-line monitoring of voltage stability is a necessary condition. Application/ Improvements: The output of the load flow analysis is used to calculate the Index that is used to maintain the system in stable limits.
\end{abstract}

Keywords: Artificial Neural Networks (ANN), Cascade Architecture (CC), Levenberg-Marquardt (LM), Stability Index, Voltage Stability

\section{Introduction}

The ability of the system to maintain voltage levels in stable both in normal conditions as well as after subjected to any kind of disturbance can be termed as voltage stability ${ }^{1}$. In recent times, there is fast growth in the increase of load demand. As the demand keeps on increasing than the actual generation, system voltage faces instability condition which causes voltage collapse ${ }^{2}$. To maintain voltage stability, Voltage Stability Margin (VSM) is calculated. VSM can be defined as the distance between the present operating point to the maximum voltage stability limit. From Figure 1, the VSM can be observed ${ }^{3,4}$. To calculate voltage stability, stability index has to be defined. Thereare many stability indices, in this paper L-Index is used ${ }^{5}$. The inputs to the L-Index are obtained from the outputs of the load flow analysis. The load flow analysis is carried out using NR method. An IEEE 30 bus system is considered in this paper ${ }^{6,7}$. Load flow analysis is done using ANN and the obtained results are compared with the outputs of the conventional method used. The error between the two methods can be observed from the graphs shown in later sections of the paper.

To calculate the load flow analysis using ANN, many architectures are available. In this paper cascade architecture (CC) with Levenberg-Marquardt (LM) algorithm is used. The Number of hidden layers is selected by using trial and error method $\stackrel{8}{ }$.

\section{Voltage Stability Index}

The voltage stability index is used to define the stability limits of the system. There are many stability indices available. Some of the stability indices are listed.

- L-Index.

- Line stability index.

- Fast voltage stability index.

- Line voltage stability index.

- Voltage Collapse prediction index.

${ }^{*}$ Author for correspondence 


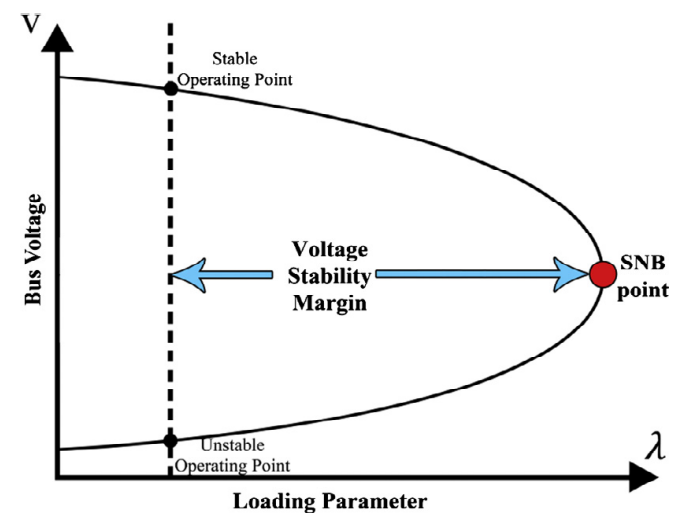

Figure 1. Voltage Stability Margin (VSM).

In this paper, the L-Index is used. The inputs to the index are obtained from the output of the load flow analysis ${ }^{9}$. To calculate the L-Index, separate the buses in the system into two groups. All load buses are brought to head and denoted as L while the PV buses are brought to tail and denoted as G.

A hybrid system equation can be formed as

$$
\left[\begin{array}{l}
V^{L} \\
I^{G}
\end{array}\right]=\left[\begin{array}{ll}
Z^{L L} & F^{L G} \\
K^{G L} & Y^{G G}
\end{array}\right]\left[\begin{array}{l}
I^{L} \\
V^{G}
\end{array}\right]
$$

Where $\mathrm{Z}^{\mathrm{LL}}, \mathrm{F}^{\mathrm{LG}}, \mathrm{K}^{\mathrm{GL}}, \mathrm{Y}^{\mathrm{GG}}$ are the sub-block of matrix $\mathrm{H}$

$\mathrm{V}^{\mathrm{L}}, \mathrm{I}^{\mathrm{G}}, \mathrm{I}^{\mathrm{L}}, \mathrm{V}^{\mathrm{G}}$ are the voltages and currents of the PV and load buses.

Voltage stability of any load bus in the system can be defined by

$$
L_{j}=\left|1-\sum_{i=1}^{g} F_{j i} \frac{V_{i}}{V_{j}}\right|
$$

The maximum limit of L-Index is set as unity. If the L-Index of any bus is beyond unity the system is said to be in unstable condition. The range of L-Index is given.

$$
R=\{L \mid 0 \leq L \leq 1\}
$$

\section{Artificial Neural Networks}

An Artificial Neural Network (ANN) is a processing network, which receives an input signal, process them and send output signals. Initially the ANNs undergo learning or training process, which is adjusting the weight co-efficient to full-fill the output conditions $\mathrm{s}^{\frac{10,11}{1}}$. There are two types of training process, supervised and unsupervised. In supervised training process the desired output is known to the neural network and adjustment of weights is done accordingly, while in unsupervised training process the desired output is unknown to the neural network ${ }^{12}$. There are different types of neural network architectures available, such as Single Layer Feed Forward Network (SLFF), Multi-Layer Feed Forward Network (MLFF), and Cascade Correlation architecture (CC). Network with an input layer, one or more hidden layers and an output layer are called as feed forward networks. In feed forward networks the signal moves only in one direction. Network with only one hidden layer is known as SLFF, while a network with a multiple number of hidden layers is called as MLFF ${ }^{13}$. In CC architecture, a neuron receives system inputs and all the outputs of the preceding layers. There are several advantages of using CC over feed forward networks, such as the learning process is quick, the network determines its own size and topology and requires no back propagation of error signals.

\section{Result and Discussion}

In this paper, an IEEE 30-bus system is considered for assessment of voltage stability. Load-flow analysis is carried out using Newton-Raphson method. The output of the load-flow analysis is used for calculating the L-Index of the system, which defines the voltage stability of the system. The calculation of L-Index is discussed in the previous sections of this paper. The system is said to be in the unstable state if the L-Index at any bus is greater than unity. For the base case study of the considered IEEE 30-bus system the L-Index at each is bus is calculated and tabulated in Table 1. Consider the following cases,

Case 1: Base Case.

Case 2: Increase $\mathrm{P}_{\mathrm{d}}$ at bus 14 alone.

Case 3: Increase $P_{d}$ at bus 19 alone.

Case 4: Increase $P_{d}$ at bus 21 alone.

Case 5: Increase $Q_{d}$ at bus 7 alone.

Hence in real-time, the system parameters are not constant and are variable time to time, contingency analysis should also be considered. The active and reactive power demand $P_{d}$ and $Q_{d}$ are increased in steps at each bus and L-Index is calculated again. This increment of $P_{d}$ and $Q_{d}$ is done till L-Index at the bus cross the unity. This increment is carried out to check for the limits of $P_{d}$ and $Q_{d}$ to which the system can hold its stability.

Considering the draw backs of using the conventional methods, the load flow analysis is carried out using ANN. 
Table 1. Summary of L-Index at different cases for an IEEE 30 bus system

\begin{tabular}{|c|c|c|c|c|c|}
\hline BUS & Case 1 & Case 2 & Case 3 & Case 4 & Case 5 \\
\hline 3 & 0.0156 & 0.0411 & 0.0363 & 0.0506 & 0.0712 \\
\hline 4 & 0.0179 & 0.0492 & 0.0433 & 0.0609 & 0.087 \\
\hline 6 & 0.016 & 0.035 & 0.0385 & 0.0618 & 0.1287 \\
\hline 7 & 0.0223 & 0.0338 & 0.0359 & 0.0495 & 1.0031 \\
\hline 9 & 0.0421 & 0.0968 & 0.1415 & 0.2756 & 0.0872 \\
\hline 10 & 0.0798 & 0.1883 & 0.2902 & 0.6079 & 0.1256 \\
\hline 12 & 0.0508 & 0.2485 & 0.1592 & 0.1842 & 0.075 \\
\hline 14 & 0.0744 & 1.0044 & 0.2471 & 0.2607 & 0.1042 \\
\hline 15 & 0.0799 & 0.4045 & 0.3185 & 0.3178 & 0.1133 \\
\hline 16 & 0.0712 & 0.2342 & 0.223 & 0.3569 & 0.1058 \\
\hline 17 & 0.0837 & 0.2101 & 0.279 & 0.5422 & 0.1276 \\
\hline 18 & 0.0983 & 0.3514 & 0.7194 & 0.4469 & 0.1385 \\
\hline 19 & 0.1044 & 0.313 & 1.006 & 0.5212 & 0.1482 \\
\hline 20 & 0.0996 & 0.2819 & 0.794 & 0.5456 & 0.144 \\
\hline 21 & 0.0944 & 0.2143 & 0.3082 & 1.0003 & 0.1443 \\
\hline 22 & 0.0938 & 0.2161 & 0.3059 & 0.9154 & 0.1434 \\
\hline 23 & 0.0958 & 0.3526 & 0.3206 & 0.4621 & 0.1383 \\
\hline 24 & 0.1066 & 0.2683 & 0.3062 & 0.6655 & 0.16 \\
\hline 25 & 0.1036 & 0.2099 & 0.2337 & 0.4468 & 0.1633 \\
\hline 26 & 0.1222 & 0.2332 & 0.2582 & 0.4831 & 0.1892 \\
\hline 27 & 0.0927 & 0.1659 & 0.1819 & 0.3172 & 0.1523 \\
\hline 28 & 0.0215 & 0.0425 & 0.0466 & 0.0761 & 0.103 \\
\hline 29 & 0.1231 & 0.2009 & 0.2178 & 0.3634 & 0.1897 \\
\hline 30 & 0.1437 & 0.2246 & 0.2421 & 0.3945 & 0.2144 \\
\hline
\end{tabular}
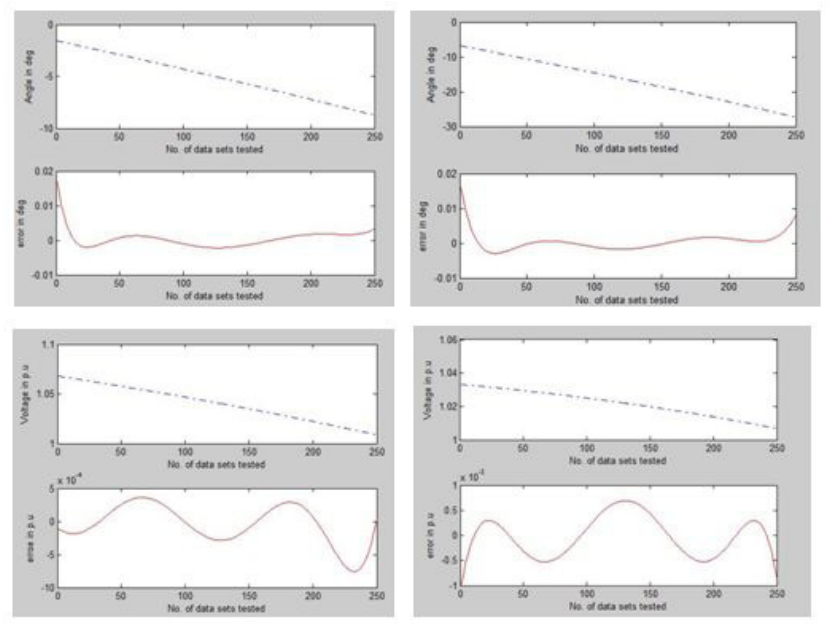

Figure 2. Comparison of conventional and ANN loadflow results at various buses.
A Cascade-Correlation architecture with LevenbergMarquardt algorithm is used to calculate the load-flow of the IEEE 30-bus system and the results are compared with the conventional method. The error between the ANN output and the conventional output are shown in Figure 2.

The shown Figure describes the voltage and angles obtained from the load-flow results and the comparison of conventional and ANN method. Since it is clearly shown that the compared results show the error close to zero, the ANN results can be used for the calculation of L-Index.

\section{Conclusion}

Since voltage stability of a system becomes major concern, on-line monitoring of the system is essential. In this paper, the voltage stability of an IEEE30 bus system is monitored in both normal and contingency conditions. This paper deals with calculating load-flow studies using ANN to overcome the drawbacks faced in using the conventional methods and the output of the load-flow studies is used to monitor voltage stability limits using the proposed Index. The results obtained in conventional and ANN are tabulated in above sections and compared. The contingency analysis is carried out to monitor the system in real-time operation.

\section{References}

1. Stevenson WD. Elements of power system analysis. 4th ed. McGraw-Hill; 1982.

2. Wood AJ, Wollenberg BF. Power generation operation and control. Newyork: Wiley; 1984.

3. Kumari MS, Maheswarapu S. Enhanced genetic algorithm based computation technique for multi-objective optimal power flow solution. International Journal of Electrical Power and Energy Systems. 2010 Jul; 32(6):736-42.

4. Pandya KS, Joshi SK. A survey of optimal power flow methods. Journal of Theoretical and Applied Information Technology. 2008 Jan; 450-8.

5. Boopathi CS, Venkadesan A, Subhransu SD. Comparison of various learning algorithms for artificial neural network based on-load flow analysis. International Review on Modelling and Simulation. 2014 Apr; 7(2):323-30.

6. Boopathi CS, Dash SS, Selvakumar K, Venkadesan A, Subramani C, Vamsikrishna D. Unit commitment problem with $\mathrm{POZ}$ constraint using dynamic programming method. International Review of Electrical Engineering. 2014 Jan; 9(1):218-25. 
7. Selvakumar K, Vijayakumar K, Palanisamy R, Karthikeyan D, Santhoshkumar G. SFLA to solve short term thermal unit commitment problem with startup and shutdown ramp limits. IREMOS. 2015 Dec; 8(6):670-8.

8. A brief description of the levenberg-marquardt algorithm implemented. Available from: http://users.ics.forth. gr/ lourakis/levmar/levmar.pdf

9. Fahlman SE, Lebiere C. The Cascade-Correlation Learning Architecture. Advances in Neural Information Processing Systems.1990; 524-32.

10. Jahromi MZ, Bioki MMH, Rashidinejad M, Fadaeinedjad R. Solution to the unit commitment problem using an artificial neural network. Turkish Journal of Electrical Engineering and Computer Sciences. 2013 Jan; 21:198-212.

11. Hsu YY, Yang CC. Fast voltage estimation using an artificial neural network. Electric Power system Research. 1993 May; 27(1):1-9.

12. Paucar VL, Rider MJ. Artificial neural networks for solving the power flow problem in electric power system. Electric Power Systems Research. 2002 Jun; 62(2):139-44.

13. Krishna J, Srivastava L. Counter propagation neural network for solving power flow problem. World Academy of Science, Engineering and Technology International Journal of Electrical, Computer, Energetic, Electronic and Communication Engineering. 2008; 2(3):521-6.

\section{Appendix}

Table A1. Bus data

\begin{tabular}{|c|c|c|c|c|c|c|c|c|}
\hline \multirow[t]{2}{*}{ Bus } & \multicolumn{2}{|c|}{ Voltage } & \multicolumn{2}{|c|}{ Generation } & \multicolumn{2}{|c|}{ Load } & \multicolumn{2}{|c|}{$\begin{array}{c}\text { Lambda } \\
\text { (\$/MVA-hr) }\end{array}$} \\
\hline & $\operatorname{Mag}(p u)$ & Ang(deg) & $\mathbf{P}(\mathbf{M W})$ & Q (MVAr) & P (MW) & Q (MVAr) & $\mathbf{P}$ & $\mathbf{Q}$ \\
\hline 1 & 1.060 & 0.000 & 212.23 & 0.00 & - & - & 36.313 & -0.128 \\
\hline 2 & 1.039 & -4.198 & 36.23 & 27.17 & 21.70 & 12.70 & 38.114 & - \\
\hline 3 & 1.021 & -6.197 & - & - & 2.40 & 1.20 & 38.823 & 0.254 \\
\hline 4 & 1.012 & -7.621 & - & - & 7.60 & 1.60 & 39.562 & 0.274 \\
\hline 5 & 1.013 & -10.825 & 29.35 & 29.95 & 94.20 & 19.00 & 40.587 & - \\
\hline 6 & 1.011 & -8.990 & - & - & - & - & 40.084 & 0.193 \\
\hline 7 & 1.004 & -10.271 & - & - & 22.80 & 10.90 & 40.570 & 0.256 \\
\hline 8 & 1.013 & -9.491 & 12.94 & 40.00 & 30.00 & 30.00 & 40.259 & 0.101 \\
\hline 9 & 1.042 & -11.806 & - & - & - & - & 40.089 & 0.146 \\
\hline 10 & 1.038 & -13.545 & - & - & 5.80 & 2.00 & 40.094 & 0.201 \\
\hline 11 & 1.060 & -11.332 & 4.40 & 9.09 & - & - & 40.088 & - \\
\hline 12 & 1.050 & -13.050 & - & - & 11.20 & 7.50 & 39.652 & 0.090 \\
\hline 13 & 1.060 & -13.050 & 0.00 & 7.73 & - & - & 39.652 & - \\
\hline 14 & 1.035 & -13.922 & - & - & 6.20 & 1.60 & 40.318 & 0.292 \\
\hline 15 & 1.031 & -13.985 & - & - & 8.20 & 2.50 & 40.507 & 0.403 \\
\hline 16 & 1.038 & -13.526 & - & - & 3.50 & 1.80 & 40.107 & 0.293 \\
\hline 17 & 1.033 & -13.757 & - & - & 9.00 & 5.80 & 40.240 & 0.323 \\
\hline 18 & 1.021 & -14.534 & - & - & 3.20 & 0.90 & 40.938 & 0.533 \\
\hline 19 & 1.019 & -14.666 & - & - & 9.50 & 3.40 & 41.033 & 0.562 \\
\hline 20 & 1.023 & -14.444 & - & - & 2.20 & 0.70 & 40.822 & 0.483 \\
\hline 21 & 1.026 & -14.005 & - & - & 17.50 & 11.20 & 40.523 & 0.456 \\
\hline 22 & 1.027 & -13.995 & - & - & - & - & 40.508 & 0.439 \\
\hline 23 & 1.021 & -14.323 & - & - & 3.20 & 1.60 & 40.879 & 0.582 \\
\hline 24 & 1.016 & -14.424 & - & - & 8.70 & 6.70 & 41.037 & 0.647 \\
\hline 25 & 1.014 & -14.003 & - & - & - & - & 40.782 & 0.712 \\
\hline 26 & 0.996 & -14.425 & - & - & 3.50 & 2.30 & 41.543 & 1.220 \\
\hline 27 & 1.022 & -13.479 & - & - & - & - & 40.319 & 0.520 \\
\hline 28 & 1.008 & -9.558 & - & - & - & - & 40.321 & 0.242 \\
\hline 29 & 1.002 & -14.713 & - & - & 2.40 & 0.90 & 41.450 & 0.837 \\
\hline 30 & 0.990 & -15.599 & - & - & 10.60 & 1.90 & 42.233 & 0.967 \\
\hline \multicolumn{3}{|c|}{ Total } & 295.14 & 113.94 & 283.40 & 126.20 & & \\
\hline
\end{tabular}


Table A2. Branch data

\begin{tabular}{|c|c|c|c|c|c|c|c|c|}
\hline \multirow{2}{*}{ Branch } & \multirow{2}{*}{ From Bus } & \multirow{2}{*}{ To Bus } & \multicolumn{2}{|c|}{ From Bus Injection } & \multicolumn{2}{|c|}{ To Bus Injection } & \multicolumn{2}{|c|}{$\operatorname{Loss}\left(I^{\wedge} 2 * Z\right)$} \\
\hline & & & $\mathbf{P}(\mathrm{MW})$ & Q(MVAr) & $\mathbf{P}(\mathrm{MW})$ & Q(MVAr) & $\mathbf{P}(\mathrm{MW})$ & Q(MVAr) \\
\hline 1 & 1 & 2 & 139.12 & -6.44 & -135.81 & 10.53 & 3.309 & 9.91 \\
\hline 2 & 1 & 3 & 73.12 & 6.44 & -70.93 & -2.89 & 2.181 & 7.97 \\
\hline 3 & 2 & 4 & 37.86 & 3.2 & -37.09 & -4.73 & 0.770 & 2.35 \\
\hline 4 & 3 & 4 & 68.53 & 1.69 & -67.94 & -0.85 & 0.595 & 1.71 \\
\hline 5 & 2 & 5 & 61.93 & 0.46 & -60.25 & 2.19 & 1.679 & 7.05 \\
\hline 6 & 2 & 6 & 50.54 & 0.28 & -49.17 & -0.03 & 1.376 & 4.18 \\
\hline 7 & 4 & 6 & 55.31 & -13.24 & -54.94 & 13.62 & 0.375 & 1.30 \\
\hline 8 & 5 & 7 & -4.60 & 8.76 & 4.65 & -10.7 & 0.053 & 0.13 \\
\hline 9 & 6 & 7 & 27.65 & -0.91 & -27.45 & -0.20 & 0.20 & 0.61 \\
\hline 10 & 6 & 8 & 18.44 & -10.64 & -18.39 & 9.90 & 0.052 & 0.18 \\
\hline 11 & 6 & 9 & 25.45 & -3.65 & -25.45 & 4.93 & 0 & 1.29 \\
\hline 12 & 6 & 10 & 15.47 & 1.5 & -15.47 & -0.26 & 0 & 1.23 \\
\hline 13 & 9 & 11 & -4.40 & -8.90 & 4.40 & 9.09 & 0 & 0.19 \\
\hline 14 & 9 & 10 & 29.85 & 3.97 & -29.85 & -3.05 & 0 & 0.92 \\
\hline 15 & 4 & 12 & 42.12 & 17.21 & -42.12 & -12.72 & 0 & 4.50 \\
\hline 16 & 12 & 13 & 0 & -7.65 & 0 & 7.73 & 0 & 0.07 \\
\hline 17 & 12 & 14 & 7.64 & 2.46 & -7.57 & -2.31 & 0.072 & 0.15 \\
\hline 18 & 12 & 15 & 17.03 & 6.87 & -16.83 & -6.47 & 0.203 & 0.40 \\
\hline 19 & 12 & 16 & 6.24 & 3.54 & -6.2 & -3.45 & 0.044 & 0.09 \\
\hline 20 & 14 & 15 & 1.37 & 0.71 & -1.37 & -0.70 & 0.005 & 0 \\
\hline 21 & 16 & 17 & 2.70 & 1.65 & -2.69 & -1.63 & 0.005 & 0.02 \\
\hline 22 & 15 & 18 & 5.49 & 1.79 & -5.46 & -1.72 & 0.034 & 0.07 \\
\hline 23 & 18 & 19 & 2.26 & 0.82 & -2.26 & -0.81 & 0.004 & 0.01 \\
\hline 24 & 19 & 20 & -7.24 & -2.59 & 7.26 & 2.63 & 0.019 & 0.04 \\
\hline 25 & 10 & 20 & 9.55 & 3.53 & -9.46 & -3.33 & 0.09 & 0.20 \\
\hline 26 & 10 & 17 & 6.32 & 4.21 & -6.31 & -4.17 & 0.017 & 0.05 \\
\hline 27 & 10 & 21 & 15.93 & 9.68 & -15.82 & -9.44 & 0.112 & 0.24 \\
\hline 28 & 10 & 22 & 7.71 & 4.38 & -7.66 & -4.27 & 0.053 & 0.11 \\
\hline 29 & 21 & 22 & -1.68 & -1.76 & 1.68 & 1.76 & 0.001 & 0 \\
\hline 30 & 15 & 23 & 4.50 & 2.89 & -4.48 & -2.83 & 0.027 & 0.05 \\
\hline 31 & 22 & 24 & 5.98 & 2.51 & -5.93 & -2.44 & 0.046 & 0.07 \\
\hline 32 & 23 & 24 & 1.28 & 1.23 & -1.27 & -1.22 & 0.004 & 0.01 \\
\hline 33 & 24 & 25 & -1.50 & 1.4 & 1.51 & -1.39 & 0.008 & 0.01 \\
\hline 34 & 25 & 26 & 3.54 & 2.37 & -3.50 & -2.30 & 0.045 & 0.07 \\
\hline 35 & 25 & 27 & -5.05 & -0.98 & 5.08 & 1.03 & 0.028 & 0.05 \\
\hline 36 & 28 & 27 & 18.36 & 5.72 & -18.36 & -4.37 & 0 & 1.35 \\
\hline 37 & 27 & 29 & 6.19 & 1.67 & -6.10 & -1.51 & 0.087 & 0.16 \\
\hline 38 & 27 & 30 & 7.09 & 1.66 & -6.93 & -1.36 & 0.163 & 0.31 \\
\hline 39 & 29 & 30 & 3.70 & 0.61 & -3.67 & -0.54 & 0.034 & 0.06 \\
\hline 40 & 8 & 28 & 1.32 & 0.09 & -1.32 & -4.45 & 0.004 & 0.01 \\
\hline 41 & 6 & 28 & 17.09 & 0.12 & -17.04 & -1.27 & 0.048 & 0.17 \\
\hline \multicolumn{7}{|c|}{ Total } & 11.742 & 47.31 \\
\hline
\end{tabular}

